\title{
The Effects of Repeated Sprint Training with Blood Flow Restriction on Strength, Anaerobic and Aerobic Performance in Basketball
}

\author{
Mohammed Elgammal $^{1, *}$, Ibrahim Hassan ${ }^{1}$, Nagla Eltanahi ${ }^{2}$, Heba Ibrahim ${ }^{2}$ \\ ${ }^{1}$ Faculty of Physical Education, Zagazig University, Zagazig, 44519, Sharqya, Egypt \\ ${ }^{2}$ Department of Self-development, Deanship of Preparatory Year, Imam Abdulrahman Bin Faisal University, Dammam, Saudi Arabia
}

Received October 19, 2020; Revised November 24, 2020; Accepted December 20, 2020

\section{Cite This Paper in the following Citation Styles}

(a): [1] Mohammed Elgammal, Ibrahim Hassan, Nagla Eltanahi, Heba Ibrahim, "The Effects of Repeated Sprint Training with Blood Flow Restriction on Strength, Anaerobic and Aerobic Performance in Basketball," International Journal of Human Movement and Sports Sciences, Vol. 8, No. 6, pp. 462 - 468, 2020. DOI: 10.13189/saj.2020.080619.

(b): Mohammed Elgammal, Ibrahim Hassan, Nagla Eltanahi, Heba Ibrahim (2020). The Effects of Repeated Sprint Training with Blood Flow Restriction on Strength, Anaerobic and Aerobic Performance in Basketball. International Journal of Human Movement and Sports Sciences, 8(6), 462 - 468. DOI: 10.13189/saj.2020.080619.

Copyright $\bigcirc 2020$ by authors, all rights reserved. Authors agree that this article remains permanently open access under the terms of the Creative Commons Attribution License 4.0 International License

\begin{abstract}
Repeated sprint training is usually performed in team sports, but its combination with blood flow restriction has a lake datum existing on the intense response to this type of training. This study aims to determine the acute effect of repeated sprint training in combination with blood flow restriction on the strength, anaerobic and aerobic performance in basketball. Twenty-four basketball players participated in current study were divided into two groups. They performed twelve on-court sessions; each consists of 3 sets of 8 repetitions of 20-sec and 4-min rests. Strength (1-RM bench press and half-squat), anaerobic, aerobic measurements were tested before the beginning of the study and two days followed by the training intervention period. The results presented a small increase in upper maximum strength (bench press) and anaerobic (Suicide Run) variables (ES 0.2 to 0.5 ) in (RST-BFR) group. In addition, there is a large increase in lower body maximum strength (half-squat) and aerobic capacity $\left(\mathrm{VO}_{2 \max }\right)$ variables. In comparison, the control group reported a small increase only in the aerobic capacity $\left(\mathrm{VO}_{2 \max }\right)(\mathrm{ES}=$ 0.26 ), otherwise trivial effect size was observed in other variables. The t-test reported a significant difference between both groups $(\mathrm{p}<0.05)$ after the (RST) with blood flow restriction on the lower body maximum strength (half-squat) and the aerobic capacity $\left(\mathrm{VO}_{2 \max }\right)$ measured variables.
\end{abstract}

Keywords Blood Flow Restriction, Aerobic Capacity, Intermittent Sports, Vascular Occlusion

\section{Introduction}

The concept of blood flow restriction (BFR) training involves a reducing of arterial blood flow to muscles, whilst occluding venous return [1]. The repeated sprint ability training (RST) is a major determinant of performance in intermittent team sports particularly in basketball [2-5].

Basketball is distinguished by high intensity short duration movements and interspersed by low to moderate intensity movement patterns [6]. Thus, it seems that the ability to perform intermittent high intensity actions over the game is critical for basketball players [7]. In this regard, several studies have shown that to be effective to enhance performance in team sports and increase the maximal oxygen uptake as well as mean and peak speed was during a repeated sprint test [8-10].

The (BFR) training has been shown to improve the muscle hypertrophy and promote the development of muscular strength using loads as low as $(20-30 \%)$ of a player's 1 repetition maximum (1RM) in athletes [11]. In addition, Holm et al. (2008) [12] found a favored high-load condition of strength adaptations and muscle 
size following a training intervention protocol for 12 weeks that compared $70 \%$ (1RM) and $15.5 \%$ (1RM).

The anaerobic and aerobic energy systems effect the (RSA), i.e., particularly anaerobic processes during sprinting and aerobic processes during recovery [13]. In this context, a review study of (BFR) mechanisms observed effects of exercises with (BFR) and concluded an increase in enhanced levels of muscle recruitment, heart rate, increases in elevated adrenal hormone concentrations and systemic hormones [14].

The (RST) at maximal intensity with incomplete recoveries (work to rest ratio $<1: 4$ ) elicits the early development of fatigue ( $33-35 \%$ power decrement), therefore impacting performance especially when exercising to exhaustion [15]. In particular, Ruscello et al. (2013) [16] described the work to rest ratio in basketball with $<1: 3$, therefore, the type of these exercises represents a challenge for the muscle (accumulation of metabolites) [17].

In the context of training response to the (BFR), previous studies indicated that long recovery time periods between training sessions are not required when applying (BFR) training [18, 19]. Thence, it seems that training with high intensity in combination with (BFR) could result in farther benefits.

In details, Park et al. (2010) [20] reported that walk training after 2 weeks with (BFR) was significantly improved the maximum aerobic capacity and anaerobic capacity in collegiate basketball players, and some studies mentioned to a significant improvement in $\mathrm{VO}_{2 \max }$ and muscle strength in (BFR) group due to low-intensity aerobic training $[18,20,21]$. On the other hand, some studies showed that training with (BFR) significantly improved the $\mathrm{VO}_{2 \max }$ compared to the non (BRF) group following 4 and 6 weeks of a high intensity training [22, $11]$.

Moreover, the (BFR) studies focused on strength gain investigations with sprint training alone [23]. Also, the (BFR) training was able to turn even a slow treadmill walking protocol into an effective training stimulus to elicit strength gains [24]. Likewise, previous studies have found a developed ability to perform sprints after (BFR) training compared to conventional one [25-27]. Moreover, (Behringer et al., 2017; Faiss et al., 2013; Valenzuela et al., 2019) [28-30] reported that applying sprints in blending with (BFR) increased sprint performance and strength and hypertrophy to a greater range than the regular training without BFR. In addition, the merging of (BFR) with sprint interval training enhances aerobic performance in trained athletes, although this did not translate to an enhanced exercise performance. Thus, sprint interval training alone did not induce any observable adaptation [31]. Accordingly, we expected the combination of repeated sprint training and (BFR) would be more effective.

To our knowledge, the combination of (RST) with (BFR) was never been investigated in basketball.
Moreover, Willis et al. (2018) [32] mentioned a lake datum exists on the intense response to a repeated sprint training session performed with (BFR). Therefore, this study aims to take part in this growing area of research by examining the question if repeated sprint training with blood flow restriction positively affects the strength, anaerobic and aerobic performance in basketball.

\section{Materials and Methods}

\subsection{Participants}

Twenty-four basketball players participated in the current study (mean $\pm \mathrm{SD}$ ) age $22.3 \pm 2.4$ years; weight, $81.2 \pm 4.7 \mathrm{~kg}$; height, $195.4 \pm 2.4 \mathrm{~cm}$. The participants were players at the university basketball team and highly trained for about $\sim 10$ hours per week with average of twelve years of training experience. Participants were categorized into two groups randomly, experimental training group with Kaatsu (TG; $\mathrm{n}=12)$ and control group $(\mathrm{CG} ; \mathrm{n}=12)$. Pre-tests were investigated before the training intervention for both groups and post-tests measured after the training sessions completed. All players were healthy and free from any chronic injuries. The informed consent obtained from each participant prior the volunteered in current study. Ethical approval was obtained for this study by the ethics committee of Zagazig University.

\subsection{Procedures}

\subsubsection{Training protocol and BFR procedures}

Each participant conducted 12 experimental sessions (RST-BFR and RST), All sessions were conducted at the same time of the day (i.e., on the afternoon). After a 15-min warm-up consisting of (stretching, basketball drills, squats and repeated sprints), both groups performed the RST session on a basketball court, which consisted of 3 sets of 8 maximal sprints performed 3 days/week for 4 weeks. Repetitions and sets were interspersed by 20 -sec and 4-min rests, respectively. The training session was conducted on a basketball court using a shuttle running of $15+15$ meters delimitated by cone. Participants were asked to sprint at maximal speed.

Prior the training intervention, the (TG) was familiarized to wear the elastic cuffs (Kaatsu-Master, Sato Sports Plaza, Tokyo, Japan) at the proximal region of legs. During the familiarization period, external pressure was selected for the cuff with $(100-130 \mathrm{mmHg})$ according to the participants blood pressure at resting phase as recommended by (T. Yasuda et al., 2008) [33]. The players wore the elastic cuffs around the proximal region of both legy (Kaatsu-Master, Sato Sports Plaza, Tokyo, Japan) during the RS training sessions. At the begging of training intervention, the cuffs were inflated with a pressure of $100 \mathrm{mmHg}$. The pressure continues to be 
increased by ten $\mathrm{mmHg}$ at every training session of RS training until the pressure reached $160 \mathrm{mmHg}$. The instruction consideration of cuffs inflation during this study was approved in accordance with recent studies [33-35].

The participants testing performed before the beginning of the study and two days followed by the training intervention period. The strength tests were attained first and followed by the anerobic measures on a same day session after 20 minutes rest period. The aerobic measures conducted on a separate day followed by the next day directly. Measurements started with the same time during the testing session days and after standardized warm-up consisting dynamic stretching, jogging, and different series of sprints.

\subsubsection{Test protocol}

\subsubsection{Strength measurement}

After a standardized warm-up, the players completed a strength warm-up using (10, 6, and 3 repetitions) with $50 \%, 75 \%$, and $85 \%$ intensities, respectively. The warm-up was estimated by their one repetitions maximum 1-RM recent values. In addition, subsequent the specific strength warm-up period, the players resistance was fixed at a critical value of $5 \%$ below the 1-RM and increased gradually after each successful trail until failure.

The 1-RM bench press and half-squat strength obtained based on the recorded maximum weight of participants were able to raise as described by (Chtara et al., 2008; Weiss et al., 2004) [36, 37]. The bench press exercises performed from the up position with full elbow extension, pulled to the chest level for a moment pause and pushed back to the starting position to finish the test. The subjects were not allowed to bounce the bar off to the chest, in addition, the foot and hand positions for each player determined during the familiarized period and were balanced during the all testing. The back-squat exercise with free weights was performed to allow the player to bend his knees to reach the position of half-squat that knee seems to be at 90-degree angle approximately while the barbell held over the shoulders. The rest period between each attempt for both strength tests was 3 minutes of recovery as stated by (Wisløff et al., 2004) [38].

\subsubsection{Anaerobic measurement}

The Suicide run test was used as an anerobic test measure in the current study. This test run was commonly used especially for basketball players in order to measure the anaerobic capacity [39]. The Suicide test consists of 143.3 meters sprint including several changes of direction tasks. The participants were instructed to start from a standing position behind the baseline and run to four different lines at maximal speed. The 4 lines description were near free-throw, half-court, free throw, and far baseline lines (5.8, 14, 22.2, and 28 meters), respectively. The players ran maximum back to the original baseline when they arrived at each line. The total time of the test course concluded as a record score of each player who was instructed to perform the test with maximal effort.

\subsubsection{Aerobic measurement}

The aerobic capacity was measured using the $20 \mathrm{~m}$ shuttle run test that consisted of $20-\mathrm{m}$ shuttle runs between two lines marked and placed twenty meters apart. The test was performed at increasing velocities, while beginning with speed of $8.5 \mathrm{~km} \cdot \mathrm{h}-1$ and maintained for 1 $\min$, thereafter the velocity was increased by $0.5 \mathrm{~km} \cdot \mathrm{h}-1$ for every minute with $10 \mathrm{~s}$ recovery period between shuttle runs until the exhaustion. The test score (total number of $20 \mathrm{~m}$ completed laps) concluded when the participants failed to complete the $20 \mathrm{~m}$ apart between the two lines in time twice or unsuccessful on two consecutive signals to arrive within 3 meters of the end line. The estimated $\mathrm{VO}_{2 \max }$ was obtained according to the final score and derived by the formula $(\mathrm{Y}=6.0 \mathrm{X}-24.4$, where $\mathrm{Y}$ equals the predicted $\mathrm{VO}_{2 \max }$ and $\mathrm{X}$ equals the maximum velocity that achieved). The reliability and validity indicator have been shown (St Clair Gibson et al., 1998) [40]. Also, this test was considered as the familiarized test of aerobic power of basketball players $[41,10]$.

\subsection{Statistical Analysis}

The descriptive data were presented as mean \pm SD and the (Shapiro-Wilk test) was used for the normality distribution examination. The homogeneity of variances was examined by using Levene's test. An independent t-test was used to measure the intragroup changes for collected data after the training intervention applied. The statistical analysis was conducted using the (SPSS 23, USA) and the significance level was applied by $\alpha=0.05$.

\section{Results}

The (mean \pm SD) of all data variables in pre and post tests are demonstrated in (Table. 1). The results demonstrate the effect size rate and the significant difference between both groups in the post-test after the intervention training period.

The TG group demonstrates a small increase in upper maximum strength (bench press) and anaerobic (Suicide Run) variables (ES 0.2 to 0.5 ). and a large increase in lower body maximum strength (half-squat) and aerobic capacity $\left(\mathrm{VO}_{2 \max }\right)$ variables. In comparison, the $\mathrm{CG}$ group demonstrates a small increase only in the aerobic capacity $\left(\mathrm{VO}_{2 \max }\right)(\mathrm{ES}=0.26)$, otherwise trivial effect size is observed in other variables. The t-test confirmed a significant difference between both groups $(p<0.05)$ after the (RST) on the lower body maximum strength (half-squat) and the aerobic capacity $\left(\mathrm{VO}_{2 \max }\right)$ measured variables. 
Table 1. The mean \pm SD and observed changes (mean $\pm 95 \% \mathrm{CI}$ ) values of measured variables at pre and post training intervention of BFR and Control groups

\begin{tabular}{|c|c|c|c|c|c|c|c|c|c|}
\hline & \multicolumn{3}{|c|}{ (BFR) Group } & \multicolumn{3}{|c|}{ (RST) Group } & \multicolumn{3}{|c|}{ (BFR - RST) Groups diff } \\
\hline & Pre-Test & Post-Test & Changes & Pre-Test & Post-Test & Changes & \multirow{2}{*}{$\begin{array}{l}\text { mean }( \pm \\
95 \% \mathrm{CI})\end{array}$} & \multirow{2}{*}{$\begin{array}{c}\mathrm{p} \\
\text { value }\end{array}$} & \multirow{2}{*}{$\begin{array}{c}\text { Effect } \\
\text { size }\end{array}$} \\
\hline & $\begin{array}{c}\text { mean } \pm \\
\text { SD }\end{array}$ & mean $\pm \mathrm{SD}$ & $\begin{array}{c}( \pm 95 \% \\
\mathrm{CI}) \\
\end{array}$ & mean $\pm \mathrm{SD}$ & mean $\pm \mathrm{SD}$ & $\begin{array}{c} \pm 95 \% \\
\mathrm{CI})\end{array}$ & & & \\
\hline \multicolumn{10}{|c|}{ Strength variables } \\
\hline $\begin{array}{l}\text { 1RM bench press } \\
(\mathrm{kg})\end{array}$ & $76.5 \pm 6.0$ & $87.3 \pm 6.9$ & $10.8 \pm 1.8$ & $74.8 \pm 5.5$ & $82.1 \pm 6.6$ & $7.3 \pm 1.3$ & $5.2 \pm 6.3$ & 0.07 & 0.78 \\
\hline $\begin{array}{l}\text { 1RM half-squat } \\
(\mathrm{kg})\end{array}$ & $\begin{array}{c}127.5 \pm \\
10.1\end{array}$ & $\begin{array}{c}150.1 \pm \\
10.6\end{array}$ & $22.7 \pm 3.0$ & $\begin{array}{c}126.0 \pm \\
7.3\end{array}$ & $\begin{array}{c}140.4 \pm \\
7.5\end{array}$ & $14.4 \pm 0.7$ & $9.8 \pm 8.1$ & 0.02 & 1.09 \\
\hline \multicolumn{10}{|c|}{ Anaerobic variables } \\
\hline Suicide Run (Sec) & $32.3 \pm 0.7$ & $32.1 \pm 0.8$ & $-0.3 \pm 0.1$ & $32.5 \pm 0.6$ & $32.3 \pm 0.6$ & $-0.2 \pm 0.1$ & $-0.3 \pm 0.7$ & 0.25 & 0.49 \\
\hline \multicolumn{10}{|c|}{ Aerobic variables } \\
\hline $\begin{array}{c}20-\mathrm{m} \text { shuttle run } \\
\text { test } \\
(\mathrm{ml} \text { kg-1 } \mathrm{min}-1)\end{array}$ & $38.3 \pm 2.2$ & $46.2 \pm 3.1$ & $7.9 \pm 2.5$ & $38.1 \pm 1.5$ & $43.9 \pm 2.9$ & $5.7 \pm 1.9$ & $2.3 \pm 1.5$ & 0.04 & 0.79 \\
\hline
\end{tabular}

\section{Discussion}

The purpose of current study was to investigate the effects of 4 weeks (RST) with (BFR) protocol. The important results were that the merging of this training appears to increase the performance of lower body strength and the aerobic capacity for the training group when compared to the control group that did the training without the practical blood occlusion. Our findings indicate an increase of the lower body strength between both groups $(\mathrm{ES} \sim 0.85)$ after the training intervention of (RST) with (BFR). In addition, the greater increase in (1RM) half-squat in TG group was higher than the CG group with significant difference $(\mathrm{p}=0.02)$ and estimated a medium rate of effect size.

There are some previous studies reported an increase of muscle strength with (BFR) training while short-term low intensity used [21], or during walking training with (BFR) $[18,24]$. The strength gain mechanism as a response after the training with (BFR) is not till now fully unspoken. (T. Abe et al., 2006) [24] reported increases in CSA at the thigh muscles after 3 weeks twice daily walking with (BFR) training. This argument explains that (BFR) is related closely to the release of metabolic that products could recruit more fast-twitch motor units to produce force, and this process induced a favorable milieu for improving anaerobic power and muscle strength [42].

In current study, the percentage improvement in (1RM) bench press strength was not larger when compared to the (1RM) half-squat that increased with higher effect size between TG and CG groups. In this regard, a percent increase in (1RM) strength is not larger than that of increase in muscle size. Recent studies reported that relative strength of elbow flexor and knee extensor muscle didn't change significantly between pre and post training intervention after low intensity (BFR) training.[24],[43, 44].

It is important to note that (RST) with (BFR) appears as feasible 'hypoxic' methods that can be performed during on-court sessions containing basketball specific movements. Previous studies had confirmed the feasibility and effectiveness of (RST) with (BFR) in racket sports [29]. Moreover, studies using (RST) with (BFR) to date had been conducted under laboratory conditions [32].

No significant difference between TG and CG groups was observed for the suicide run test as anaerobic test. These results indicate that anaerobic performance is not affected by occlusion during the (RST) with (BFR) in basketball [45].

These results showed like previous investigation that reported in Australian junior players of national level before the institution of the new rules [46]. Many authors and coaches use distances of 20 or $25 \mathrm{~m}$ to test their players because it is close to the length of a basketball court [47]. However, during the game, players are rarely in a situation where they have to sprint on the whole court distance, and video analysis of competitions has shown that the high-intensity runs performed by players of national level lasted between 1.7 and 2.1 seconds, which almost corresponds to distances of 10 meter [48]. In current study, no significant increases in anaerobic performance were observed when used the (RST) with (BFR). These results conflicted to those reported in response to training with (BFR) [49, 50]. Accordingly, it seems no relationship between the volume of (BFR) with (RST) and improvements in anaerobic performance.

In addition, the results indicated an influence of combining (RST) with (BFR) in increasing $\mathrm{VO}_{2 \max }$ in basketball players. The present study presents preliminary evidence to suggest that enhanced aerobic performance could occur with (BFR) compared with (RST) alone. Also, the $\mathrm{VO}_{2 \max }$ increased by $4.5 \%$ in response to (RST) with (BFR), compared with $0.7 \%$ in (RST) alone. Enhancing oxygen uptake to other forms of exercise has been demonstrated previously as influence of combining (BFR) $[18,20]$. 
We attempted to identify an optimal combination of (BFR) and exercise intensity to maximize aerobic performance. In this context, the results showed a greater improvement in aerobic in response to complete (RST) with (BFR). This improvement in $\mathrm{VO}_{2 \max }$ agrees with those studies that investigated the effect of (RST) exercises in combination with (BFR), while cuff pressure was applied at 140 to $180 \mathrm{mHg}$ [51, 52]. [30]. these findings consisted with reported benefits of (BFR) training with moderate intensity on the aerobic performance [53] and gave an evidence for several contributions to this mechanism.

\section{Conclusion}

We concluded that merging repeated sprint training with (BFR) might alter muscular strength and aerobic performance in basketball players, but no noticeable change was observed to the anerobic performance. In both study groups, the results showed an improvement in all variables with a trend for greater increases in lower body strength in response to (RST) with (BFR) training and applied occlusion with pressure reached to $160 \mathrm{mmHg}$.

\section{Acknowledgement}

The authors are grateful to the players of basketball team for their participation and cooperation in this study.

\section{Conflicts of Interest}

The authors declare no conflict of interest.

\section{REFERENCES}

[1] Pope, Zachary, Jeffrey Willardson, and Brad Schoenfeld, "A Brief Review: Exercise and Blood Flow Restriction," The Journal of Strength and Conditioning Research, vol. 27, pp. 2914-2926, 10/01, 2013.

[2] Castagna, C., G. Abt, V. Manzi, G. Annino, E. Padua, and S. D'Ottavio, "Effect of recovery mode on repeated sprint ability in young basketball players," J Strength Cond Res, vol. 22, no. 3, pp. 923-9, May, 2008.

[3] Castagna, C., V. Manzi, S. D'Ottavio, G. Annino, E. Padua, and D. Bishop, "Relation between maximal aerobic power and the ability to repeat sprints in young basketball players," J Strength Cond Res, vol. 21, no. 4, pp. 1172-6, Nov, 2007.

[4] Girard, O., A. Mendez-Villanueva, and D. Bishop, "Repeated-sprint ability - part I: factors contributing to fatigue," Sports Med, vol. 41, no. 8, pp. 673-94, Aug 1, 2011.

[5] McGawley, K., and D. Bishop, "Reliability of a 5 x 6-s maximal cycling repeated-sprint test in trained female team-sport athletes," Eur J Appl Physiol, vol. 98, no. 4, pp. 383-93, Nov, 2006

[6] Padulo, J., G. Attene, G. M. Migliaccio, F. Cuzzolin, S. Vando, and L. P. Ardigo, "Metabolic optimisation of the basketball free throw," J Sports Sci, vol. 33, no. 14, pp. $1454-8,2015$.

[7] Ben Abdelkrim, N., S. El Fazaa, and J. El Ati, "Time-motion analysis and physiological data of elite under-19-year-old basketball players during competition," Br J Sports Med, vol. 41, no. 2, pp. 69-75; discussion 75, Feb, 2007.

[8] Bishop, D., O. Girard, and A. Mendez-Villanueva, "Repeated-sprint ability - part II: recommendations for training," Sports Med, vol. 41, no. 9, pp. 741-56, Sep 1, 2011.

[9] Hunter, J. R., B. J. O'Brien, M. G. Mooney, J. Berry, W. B. Young, and N. Down, "Repeated sprint training improves intermittent peak running speed in team-sport athletes," $J$ Strength Cond Res, vol. 25, no. 5, pp. 1318-25, May, 2011.

[10] Meckel, Y., R. Gottlieb, and A. Eliakim, "Repeated sprint tests in young basketball players at different game stages," Eur J Appl Physiol, vol. 107, no. 3, pp. 273-9, Oct, 2009.

[11] Paton, Carl D, Shalako M Addis, and Lee-Anne Taylor, "The effects of muscle blood flow restriction during running training on measures of aerobic capacity and run time to exhaustion," European journal of applied physiology, vol. 117 , no. 12, pp. 2579-2585, 2017.

[12] Holm, L., S. Reitelseder, T. G. Pedersen, S. Doessing, S. G. Petersen, A. Flyvbjerg, J. L. Andersen, P. Aagaard, and M. Kjaer, "Changes in muscle size and MHC composition in response to resistance exercise with heavy and light loading intensity," J Appl Physiol (1985), vol. 105, no. 5, pp. 1454-61, Nov, 2008.

[13] Spencer, M., D. Bishop, B. Dawson, and C. Goodman, "Physiological and metabolic responses of repeated-sprint activities:specific to field-based team sports," Sports Med, vol. 35, no. 12, pp. 1025-44, 2005.

[14] Loenneke, Jeremy Paul, Thomas Joseph Pujol, and Conditioning Journal, "The use of occlusion training to produce muscle hypertrophy," J Strength, vol. 31, no. 3, pp. 77-84, 2009.

[15] Bishop, D., J. Edge, C. Davis, and C. Goodman, "Induced metabolic alkalosis affects muscle metabolism and repeated-sprint ability," Med Sci Sports Exerc, vol. 36, no. 5, pp. 807-13, May, 2004.

[16] Ruscello, B., N. Tozzo, G. Briotti, E. Padua, F. Ponzetti, and S. D'Ottavio, "Influence of the number of trials and the exercise to rest ratio in repeated sprint ability, with changes of direction and orientation," J Strength Cond Res, vol. 27, no. 7, pp. 1904-19, Jul, 2013.

[17] Spriet, L. L., M. I. Lindinger, R. S. McKelvie, G. J. Heigenhauser, and N. L. Jones, "Muscle glycogenolysis and $\mathrm{H}+$ concentration during maximal intermittent cycling," $J$ Appl Physiol (1985), vol. 66, no. 1, pp. 8-13, Jan, 1989.

[18] Abe, T, T Yasuda, T Midorikawa, Y Sato, K Inoue, K Koizumi, and N Ishii, "Skeletal muscle size and circulating IGF-1 are increased after two weeks of twice daily 
"KAATSU" resistance training," International Journal of KAATSU Training Research, vol. 1, no. 1, pp. 6-12, 2005.

[19] Yasuda, Tomohiro, William F Brechue, Taku Fujita, Jun Shirakawa, Yoshiaki Sato, and Takashi Abe, "Muscle activation during low-intensity muscle contractions with restricted blood flow," Journal of sports sciences, vol. 27, no. 5, pp. 479-489, 2009.

[20] Park, S., J. K. Kim, H. M. Choi, H. G. Kim, M. D. Beekley, and H. Nho, "Increase in maximal oxygen uptake following 2-week walk training with blood flow occlusion in athletes," Eur J Appl Physiol, vol. 109, no. 4, pp. 591-600, Jul, 2010.

[21] de Oliveira, Mariana Fernandes Mendes de, Fabrizio Caputo, Rogério Bulhões Corvino, Benedito Sérgio Denadai, and science in sports, "Short - term low - intensity blood flow restricted interval training improves both aerobic fitness and muscle strength," J Scandinavian journal of medicine, vol. 26, no. 9, pp. 1017-1025, 2016.

[22] Keramidas, M. E., S. N. Kounalakis, and N. D. Geladas, "The effect of interval training combined with thigh cuffs pressure on maximal and submaximal exercise performance," Clin Physiol Funct Imaging, vol. 32, no. 3, pp. 205-13, May, 2012.

[23] Markovic, G., I. Jukic, D. Milanovic, and D. Metikos, "Effects of sprint and plyometric training on muscle function and athletic performance," J Strength Cond Res, vol. 21, no. 2, pp. 543-9, May, 2007.

[24] Abe, T., C. F. Kearns, and Y. Sato, "Muscle size and strength are increased following walk training with restricted venous blood flow from the leg muscle, Kaatsu-walk training," $J$ Appl Physiol (1985), vol. 100, no. 5, pp. 1460-6, May, 2006.

[25] Cook, C. J., L. P. Kilduff, and C. M. Beaven, "Improving strength and power in trained athletes with 3 weeks of occlusion training," Int J Sports Physiol Perform, vol. 9, no. 1, pp. 166-72, Jan, 2014.

[26] Scott, B. R., J. P. Loenneke, K. M. Slattery, and B. J. Dascombe, "Blood flow restricted exercise for athletes: A review of available evidence," J Sci Med Sport, vol. 19, no. 5, pp. 360-7, May, 2016.

[27] Scott, B. R., J. J. Peiffer, and P. S. R. Goods, "The Effects of Supplementary Low-Load Blood Flow Restriction Training on Morphological and Performance-Based Adaptations in Team Sport Athletes," J Strength Cond Res, vol. 31, no. 8, pp. 2147-2154, Aug, 2017.

[28] Behringer, M., D. Behlau, J. C. K. Montag, M. L. McCourt, and J. Mester, "Low-Intensity Sprint Training With Blood Flow Restriction Imp roves 100-m Dash," J Strength Cond Res, vol. 31, no. 9, pp. 2462-2472, Sep, 2017.

[29] Faiss, R., O. Girard, and G. P. Millet, "Advancing hypoxic training in team sports: from intermittent hypoxic training to repeated sprint training in hypoxia," Br J Sports Med, vol. 47 Suppl 1, pp. i45-50, Dec, 2013.

[30] Valenzuela, P. L., G. Sanchez-Martinez, E. Torrontegi, J. Vazquez-Carrion, M. Gonzalez, Z. Montalvo, and G. P. Millet, "Acute responses to On-Court Repeated-Sprint Training Performed With Blood Flow Restriction vs Systemic Hypoxia in Elite Badminton Athletes," Int J Sports Physiol Perform, pp. 1-27, Apr 8, 2019.

[31] Taylor, C. W., S. A. Ingham, and R. A. Ferguson, “Acute and chronic effect of sprint interval training combined with postexercise blood-flow restriction in trained individuals," Exp Physiol, vol. 101, no. 1, pp. 143-54, Jan, 2016.

[32] Willis, S. J., L. Alvarez, F. Borrani, and G. P. Millet, "Oxygenation time course and neuromuscular fatigue during repeated cycling sprints with bilateral blood flow restriction," Physiol Rep, vol. 6, no. 19, pp. e13872, Sep, 2018.

[33] Yasuda, T., W. F. Brechue, T. Fujita, Y. Sato, and T. Abe, "Muscle activation during low-intensity muscle contractions with varying levels of external limb compression," J Sports Sci Med, vol. 7, no. 4, pp. 467-74, 2008.

[34] Yasuda, T., T. Abe, W. F. Brechue, H. Iida, H. Takano, K. Meguro, M. Kurano, S. Fujita, and T. Nakajima, "Venous blood gas and metabolite response to low-intensity muscle contractions with external limb compression," Metabolism, vol. 59, no. 10, pp. 1510-9, Oct, 2010.

[35] Yasuda, T., S. Fujita, R. Ogasawara, Y. Sato, and T. Abe, "Effects of low-intensity bench press training with restricted arm muscle blood flow on chest muscle hypertrophy: a pilot study," Clin Physiol Funct Imaging, vol. 30, no. 5, pp. 338-43, Sep, 2010.

[36] Chtara, M., A. Chaouachi, G. T. Levin, M. Chaouachi, K. Chamari, M. Amri, and P. B. Laursen, "Effect of concurrent endurance and circuit resistance training sequence on muscular strength and power development," J Strength Cond Res, vol. 22, no. 4, pp. 1037-45, Jul, 2008.

[37] Weiss, L. W., L. E. Wood, A. C. Fry, R. B. Kreider, G. E. Relyea, D. B. Bullen, and P. D. Grindstaff, "Strength/power augmentation subsequent to short-term training abstinence," J Strength Cond Res, vol. 18, no. 4, pp. 765-70, Nov, 2004.

[38] Wisløff, U, C Castagna, J Helgerud, R Jones, and J Hoff, "Maximal squat strength is strongly correlated to sprint-performance and vertical jump height in elite soccer players," J Br J Sports Med, vol. 38, pp. 285-288, 2004.

[39] McKeag, Douglas, Basketball, Malden, Mass.: Blackwell Science ; Oxford : Blackwell Pub., 2003.

[40] St Clair Gibson, A, S Broomhead, MI Lambert, and JA Hawley, "Prediction of maximal oxygen uptake from a 20-m shuttle run as measured directly in runners and squash players," Journal of Sports Sciences, vol. 16, no. 4, pp. 331-335, 1998.

[41] Ferioli, Davide, Ermanno Rampinini, Andrea Bosio, Antonio La Torre, Matteo Azzolini, and Aaron J Coutts, "The physical profile of adult male basketball players: Differences between competitive levels and playing positions," Journal of sports sciences, vol. 36, no. 22, pp. 2567-2574, 2018.

[42] Moore, D. R., K. A. Burgomaster, L. M. Schofield, M. J. Gibala, D. G. Sale, and S. M. Phillips, "Neuromuscular adaptations in human muscle following low intensity resistance training with vascular occlusion," Eur J Appl Physiol, vol. 92, no. 4-5, pp. 399-406, Aug, 2004.

[43] Fujita, T., William Brechue, Koji Kurita, Yasushi Sato, and T. Abe, "Increased muscle volume and strength following six days of low-intensity resistance training with restricted muscle blood flow," International Journal of Kaatsu Training Research, vol. 4, pp. 1-8, 01/01, 2008.

[44] Takarada, Y., H. Takazawa, Y. Sato, S. Takebayashi, Y. 
Tanaka, and N. Ishii, "Effects of resistance exercise combined with moderate vascular occlusion on muscular function in humans," J Appl Physiol (1985), vol. 88, no. 6, pp. 2097-106, Jun, 2000.

[45] Delextrat, A., and D. Cohen, "Physiological testing of basketball players: toward a standard evaluation of anaerobic fitness," J Strength Cond Res, vol. 22, no. 4, pp. 1066-72, Jul, 2008.

[46] Hoare, D. G., "Predicting success in junior elite basketball players--the contribution of anthropometic and physiological attributes," J Sci Med Sport, vol. 3, no. 4, pp. 391-405, Dec, 2000.

[47] Hoffman, Jay, Shmuel Epstein, Merav Einbinder, and Yitzhak Weinstein, "A Comparison Between the Wingate Anaerobic Power Test to Both Vertical Jump and Line Drill Tests in Basketball," The Journal of Strength \& Conditioning Research, vol. 14, 08/01, 2000.

[48] McInnes, S. E., J. S. Carlson, C. J. Jones, and M. J. McKenna, "The physiological load imposed on basketball players during competition," J Sports Sci, vol. 13, no. 5, pp. 387-97, Oct, 1995.

[49] Burgomaster, K. A., D. R. Moore, L. M. Schofield, S. M. Phillips, D. G. Sale, and M. J. Gibala, "Resistance training with vascular occlusion: metabolic adaptations in human muscle," Med Sci Sports Exerc, vol. 35, no. 7, pp. 1203-8, Jul, 2003.

[50] Sundberg, C. J., "Exercise and training during graded leg ischaemia in healthy man with special reference to effects on skeletal muscle," Acta Physiol Scand Suppl, vol. 615, pp. $1-50,1994$.

[51] Brechbuhl, C., F. Brocherie, G. P. Millet, and L. Schmitt, "Effects of Repeated-Sprint Training in Hypoxia on Tennis-Specific Performance in Well-Trained Players," Sports Med Int Open, vol. 2, no. 5, pp. E123-E132, Sep, 2018 .

[52] Brechbuhl, Cyril, Laurent Schmitt, Grégoire P. Millet, and Franck Brocherie, "Shock microcycle of repeated-sprint training in hypoxia and tennis performance: Case study in a rookie professional player," International Journal of Sports Science \& Coaching, vol. 13, no. 5, pp. 723-728, 2018/10/01, 2018.

[53] Amani, Ali Reza, Hassan Sadeghi, Taher Afsharnezhad, and Medicine, "Interval training with blood flow restriction on aerobic performance among young soccer players at transition phase," J Montenegrin Journal of Sports Science, vol. 7 , no. 2 , pp. 5 , 2018 